\title{
Chemotherapy-induced neutropenia/febrile neutropenia prophylaxis with biosimilar filgrastim in solid tumors versus hematological malignancies: MONITOR-GCSF study
}

\author{
Heinz Ludwig ${ }^{1}$, Carsten Bokemeyer ${ }^{2}$, Matti Aapro ${ }^{3}$, Mario Boccadoro $^{4}$, Pere Gascón ${ }^{5}$, Kris \\ Denhaerynck $^{6,7}$, Andriy Krendyukov ${ }^{8}$, Ivo Abraham*,6,9,10,11 \& Karen MacDonald 6 \\ ${ }^{1}$ Medizinische Abteilung I- Onkologie und Haematologie, Wilhelminenspital, Wienpäoh, Montleartstraße 37, 1160 Vienna, \\ Austria \\ ${ }^{2}$ Department of Oncology, Hematology \& BMT with Section of Pneumology Universitaetsklinikum Hamburg Eppendorf, \\ Martinistraße 52, 20246 Hamburg, Germany \\ ${ }^{3}$ Cancer Center, Clinique de Genolier, Route du Muids 3, 1272 Genolier, Switzerland \\ ${ }^{4}$ Dipartimento di Oncologia e Ematologia, Azienda Ospedaliero Universitaria S Giovanni Battista di Torino, Via Cherasco 15, 10126 \\ Torino, Italy \\ ${ }^{5}$ Department of Hematology-Oncology, Division of Medical Oncology, Hospital Clínic de Barcelona, University of Barcelona, Carrer \\ de Villarroel 170, 08036 Barcelona, Spain \\ ${ }^{6}$ Matrix45, Tucson, 6159 West Sunset Road, Tucson, AZ 85743, USA \\ ${ }^{7}$ Department of Public Health, University of Basel, Bernoullistrasse 28, 4056 Basel, Switzerland \\ ${ }^{8}$ Hematology and Oncology, Hexal AG, Industriestraße 25, 83607 Holzkirchen, Germany (formerly) \\ ${ }^{9}$ Department of Pharmacy Practice \& Science, College of Pharmacy, University of Arizona, Tucson, AZ 85721, USA \\ ${ }^{10}$ Center for Health Outcomes \& PharmacoEconomic Research, College of Pharmacy, University of Arizona, Tucson, AZ 85721, USA \\ ${ }^{11}$ Department of Family \& Community Medicine, College of Medicine, University of Arizona, Tucson, AZ 85724, USA \\ *Author for correspondence: Tel.: +1 520626 4425; abraham@pharmacy.arizona.edu
}

\begin{abstract}
Aim: This study aimed to report patterns of biosimilar filgrastim prophylaxis and outcomes of chemotherapy-induced neutropenia (CIN)/febrile neutropenia (FN) in patients with hematological malignancies or solid tumors. Patients \& methods: MONITOR-GCSF is a real-world study of 1447 cancer patients receiving CIN/FN prophylaxis with biosimilar filgrastim (solid tumors: $77.2 \%$; hematological malignancies: $22.8 \%)$. Results: Differences in prophylaxis intensity and day of initiation relative to guideline recommendations were observed. In hematology patients, higher rates of CIN and FN occurred at cycle level, and rate of FN was higher at patient level (9.1 vs 5.0\% in solid tumor patients). Conclusion: Adequate GCSF support in hematology and solid tumor patients is important to prevent CIN/FN and related hospitalizations and chemotherapy disturbances.
\end{abstract}

First draft submitted: 22 October 2018; Accepted for publication: 5 December 2018; Published online: 4 March 2019

Keywords: biosimilar $\bullet$ filgrastim $\bullet$ granulocyte colony-stimulating factor $\bullet$ hematological malignancies $\bullet$ neutropenia • solid tumors

Chemotherapy-induced neutropenia (CIN) and febrile neutropenia (FN) are common but potentially lifethreatening complications of myelotoxic chemotherapy, especially regimens with FN risk $\geq 20 \%$ and, for patients with risk factors, regimens with FN risk 10-20\%. CIN/FN often leads to hospitalization or disruption in chemotherapy [1-5]. Standard practice includes prophylaxis with GCSF based on the relative myelotoxicity of the regimen [6-8]. Standard and pegylated formulations of filgrastim are efficacious in preventing or reducing severity and duration of CIN/FN [4,5,9-13]. GCSF is indicated for patients with solid tumors as well as those with lymphoproliferative disorders [6-8]; however, use in other hematological settings is unclear [7,14-18]. GCSF is also recommended in patients with soft tissue sarcoma receiving high-risk regimens $[6,8]$ and those receiving dose-dense chemotherapy 
regimens in certain solid tumors (e.g., high-risk breast cancer and urothelial cancer) including pediatric patients with Ewing sarcoma [7].

The MONITOR-GCSF study [19-21] evaluated patients with solid tumors (breast, ovarian, bladder, lung or prostate) or hematological malignancies (diffuse large B-cell lymphoma [DLBCL] or multiple myeloma [MM]) receiving biosimilar filgrastim (EP-2006 [Hexal AG/Sandoz International GmbH] marketed as Zarzio ${ }^{\circledR}$ in Europe and Zarxio ${ }^{\circledR}$ in the USA). We report comparisons between these two cohorts in terms of observed prophylaxis patterns and CIN/FN outcomes. Further results of predictive modeling of outcomes for each cohort are presented. Static models used patients, and dynamic models used cycles, as the unit of analysis. Patient-level analyses modeled outcomes 'ever' experienced during the course of chemotherapy and identified determinants that can be assessed at the start of chemotherapy. Cycle-level analyses modeled outcomes observed within each chemotherapy cycle and identified determinants that should be assessed at each cycle as patients progress through chemotherapy, as recommended by the European Organisation for Research and Treatment of Cancer (EORTC) guidelines [6].

\section{Materials \& methods}

Methodology of MONITOR-GCSF, including study design, sampling, data model and statistical analysis, has been described in detail [19-22]. Elements relevant to the findings reported here are summarized.

\section{Design}

This was a prospective, real-world, observational study of cancer patients receiving chemotherapy whose physician prescribed Zarzio ${ }^{\circledR}$ (Sandoz GmbH, Kundl, Austria). Conducted in 140 centers in 12 European countries, eligible subjects were adults with prevalent cancers commonly treated with myelotoxic chemotherapy regimens including stage 3 or 4 breast, ovarian, bladder or lung cancer; metastatic prostate cancer; or stage 3 or 4 DLBCL or MM. Patients were observed for up to six cycles of chemotherapy. The study was conducted in accordance with the Declaration of Helsinki and all patients provided written informed consent.

\section{Outcomes}

Outcomes at both the patient and cycle levels included: CIN grade 4 (CIN4), FN, CIN/FN-related hospitalization and chemotherapy disturbance, and a composite index of any of these outcomes.

\section{Special indices}

Prophylaxis intensity categorized patients as under-, correctly- or over-prophylacted relative to the amended EORTC guidelines [6].

The patient risk score (PRS; range: 0-11) weighs eight CIN/FN-related patient risk factors specified in the EORTC guidelines. Developed by consensus of four of the authors (H Ludwig, C Bokemeyer, M Aapro, P Gascón), a weight of 3 was given to age $\geq 65$ and history of prior FN; weight of 1.5 to advanced disease and poor performance and/or nutritional status; and 0.5 to no antibiotic prophylaxis, female sex, hemoglobin $<12 \mathrm{~g} / \mathrm{dl}$, and renal, cardiovascular or liver disease.

The GCSF initiation score (GIS; range: $0-1)$ rates the day of Zarzio ${ }^{\circledR}$ initiation relative to chemotherapy administration. A GIS of 1 was assigned to each cycle in which Zarzio ${ }^{\circledR}$ was started within the recommended 24-72-h window following completion of chemotherapy; a score of 0 if initiated on the same day as chemotherapy $(<24 \mathrm{~h})$ or on day 10 or later; a score of 0.75 if initiated on days 4-6 and 0.50 if on days 7-9.

Advanced disease referred to stage 4 (stage 3 if $\mathrm{MM}$ ) and prior chemotherapy in the metastatic setting.

\section{Variables included in modeling}

Patient data at enrollment: age; gender; cancer type; history of CIN4, FN, repeated infections and anemia; performance status (Eastern Cooperative Oncology Group [ECOG]); advanced disease; antibiotic prophylaxis; hemoglobin; liver, cardiovascular or renal disease; number of prior lines of chemotherapy; radiotherapy; prophylaxis type; prophylaxis intensity; initial Zarzio ${ }^{\circledR}$ dose; GIS; Zarzio ${ }^{\circledR}$ duration; and PRS. Since prophylaxis intensity was derived from the guideline algorithm and based on chemotherapy-associated FN risk categories $(<10,10-20$ and $>20 \%$ ), and hence the two are correlated, chemotherapy-associated FN risk group was not included as a predictor in any of the modeling. 
Patient data at each cycle: hemoglobin; prophylactic antibiotic therapy; ECOG; weight loss >5\% in 1 month; CIN any grade (CIN1/4) in previous cycle; infection in previous cycle; Zarzio ${ }^{\circledR}$ dose; Zarzio ${ }^{\circledR}$ duration; PRS; and GIS.

Aggregate and 'ever during study' patient data: mean GIS; mean Zarzio ${ }^{\circledR}$ duration; 'ever' ECOG $\geq 2$; 'ever' CIN4; 'ever' FN; 'ever' CIN/FN-related hospitalization; 'ever' CIN/FN-related chemotherapy disturbance; 'ever' positive CIN/FN composite score.

Center data: type (academic, academic affiliated, nonacademic); case mix (cancer patients; newly diagnosed cancer patients; chemotherapy-treated patients; chemotherapy-treated patients with FN); FN prevention policy and/or protocol (sum, range: 0-2); number of guidelines used (EORTC, European Society for Medical Oncology, American Society of Medical Oncology, National Comprehensive Cancer Network and/or local; sum, range: 0-5).

Physician data: age; gender; attendance at educational event on FN prevention in past year; knowledge about FN risk factors (range: $0-10$ ).

\section{Statistical issues}

Cycle data were 'nested' under patients, and patients under centers, thus violating the statistical assumption of independence. To adjust for statistical dependence, we used generalized estimating equations [23]. Chemotherapy disturbances were measured in the cycle after a CIN/FN episode occurred (lag = 1). Multivariate logistic regression was used in both patient- and cycle-level analyses to estimate adjusted odds ratios. Predictive performance of models was tested by the c-statistic.

\section{Results}

\section{Patient cohorts}

The evaluable sample included $1117(77.2 \%)$ solid tumor patients and 330 (22.8\%) with hematological malignancies (Table 1). Solid tumors included stage 3 or 4 breast $(n=466)$, lung $(n=345)$, ovarian $(n=140)$ and bladder $(n=64)$, as well as metastatic prostate cancer $(n=102)$; hematological malignancies included stage 3 or 4 DLBCL $(n=245)$ and MM ( $=85)$. The solid tumor cohort had disproportionately more women due to the preponderance of breast cancer patients $(\mathrm{p}<0.001)$; difference in mean age was not statistically significant ( $\mathrm{p}$ $=$ n.s. [not significant]). Hematological patients had proportionately higher ECOG scores $(\mathrm{p}=0.011)$. There was no difference between group means on the weighted PRS index ( $\mathrm{p}=\mathrm{n} . \mathrm{s}$.); however, differences in some of the unweighted EORTC-listed FN risk factors were found.

The cohorts differed in distribution of chemotoxicity $(\mathrm{p}<0.001)$. About half $(50.9 \%)$ with solid tumors had a regimen with $10-20 \% \mathrm{FN}$ risk and about a third with $\geq 20 \%$ risk. Although over $70 \%$ of hematology patients had regimens with $\geq 20 \% \mathrm{FN}$ risk, this was mainly among DLBCL patients ( 90 vs $18 \%$ in $\mathrm{MM}$ ).

\section{Prophylaxis patterns}

Zarzio ${ }^{\circledR}$ treatment patterns are shown in Table 2. The solid tumor cohort included significantly $(\mathrm{p}=0.009)$ more over-prophylacted patients and fewer correctly-prophylacted patients than the hematology cohort (Figure 1). Note, $16.3 \%$ of those with solid tumors and $21.0 \%$ of hematology patients were under-prophylacted. Mean \pm standard deviation day of GCSF initiation after chemotherapy was $2.60 \pm 2.70$ for those with solid tumors and $4.76 \pm 3.30$ for hematology patients ( $\mathrm{p}<0.001$ ). In $60.0 \%$ of solid tumor patients, Zarzio ${ }^{\circledR}$ was initiated in the recommended day 1-3 (24-72-h) window, but in over half of hematology patients (53.8\%), initiation occurred late.

\section{Clinical outcomes}

In the patient-level ('ever' during study) analyses there were no significant differences between cohorts in rates of CIN episodes, hospitalization or chemotherapy disturbance, or composite outcome ( all $\mathrm{p}=\mathrm{n} . \mathrm{s}$.); except that in $9.1 \%$ of hematology patients but only $5.0 \%$ of solid tumor patients an FN episode occurred ( $p=0.032$; Figure 2). In contrast, the cycle-level analyses revealed a proportionately higher incidence of CIN1/4, grade 3 or 4 CIN (CIN3/4) and CIN4 in the hematology cohort, as well as a higher rate of in-cycle FN (all p $<0.05$ ).

\section{Predictive risk modeling: patients with solid tumors}

Patient-level analysis

The risk of a solid tumor patient 'ever' experiencing CIN4 during the study increased with baseline history of CIN4; liver, renal or cardiac disease; or concomitant antibiotic prophylaxis (Supplementary Tables 1 Ai \& 2). FN 
Table 1. Patient demographics and clinical status; cancer and chemotherapy-induced neutropenia/febrile neutropenia history and management.

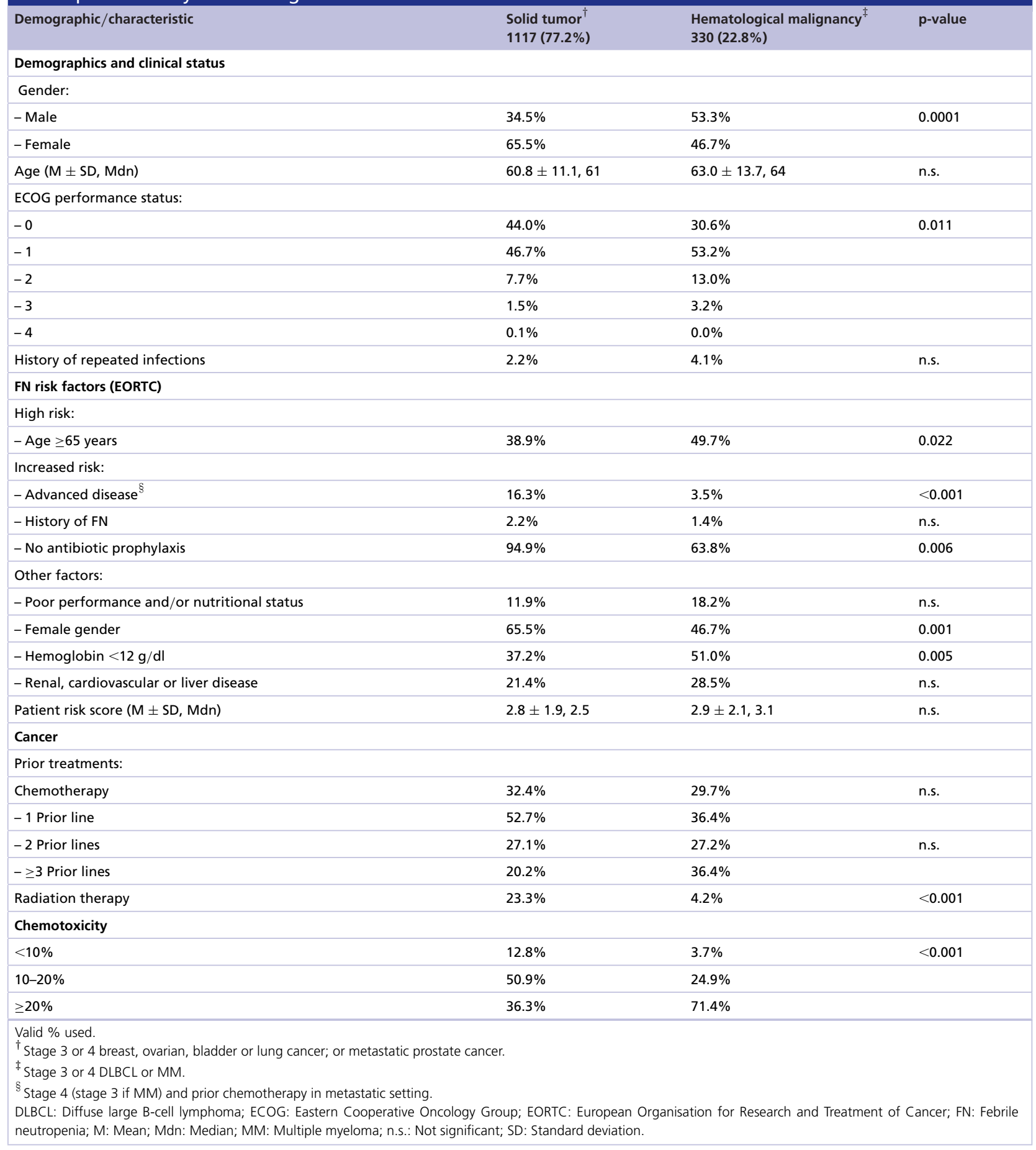

risk increased with ECOG score $\geq 2$ anytime during the study. Risk of ever having a CIN/FN-related hospitalization rose with ECOG $\geq 2$ and under-prophylaxis. Odds of CIN/FN-related chemotherapy disturbance increased with female sex; liver, renal or cardiac disease; and secondary prophylaxis. A score of 1 on the composite outcome rose with female sex; baseline history of CIN4; liver, renal or cardiac disease; concomitant antibiotic prophylaxis; and under-prophylaxis. Reduced risks of CIN4, FN, hospitalization and the composite outcome were associated with over-prophylaxis. The c-statistics for these models ranged from 0.64 to 0.70 . 


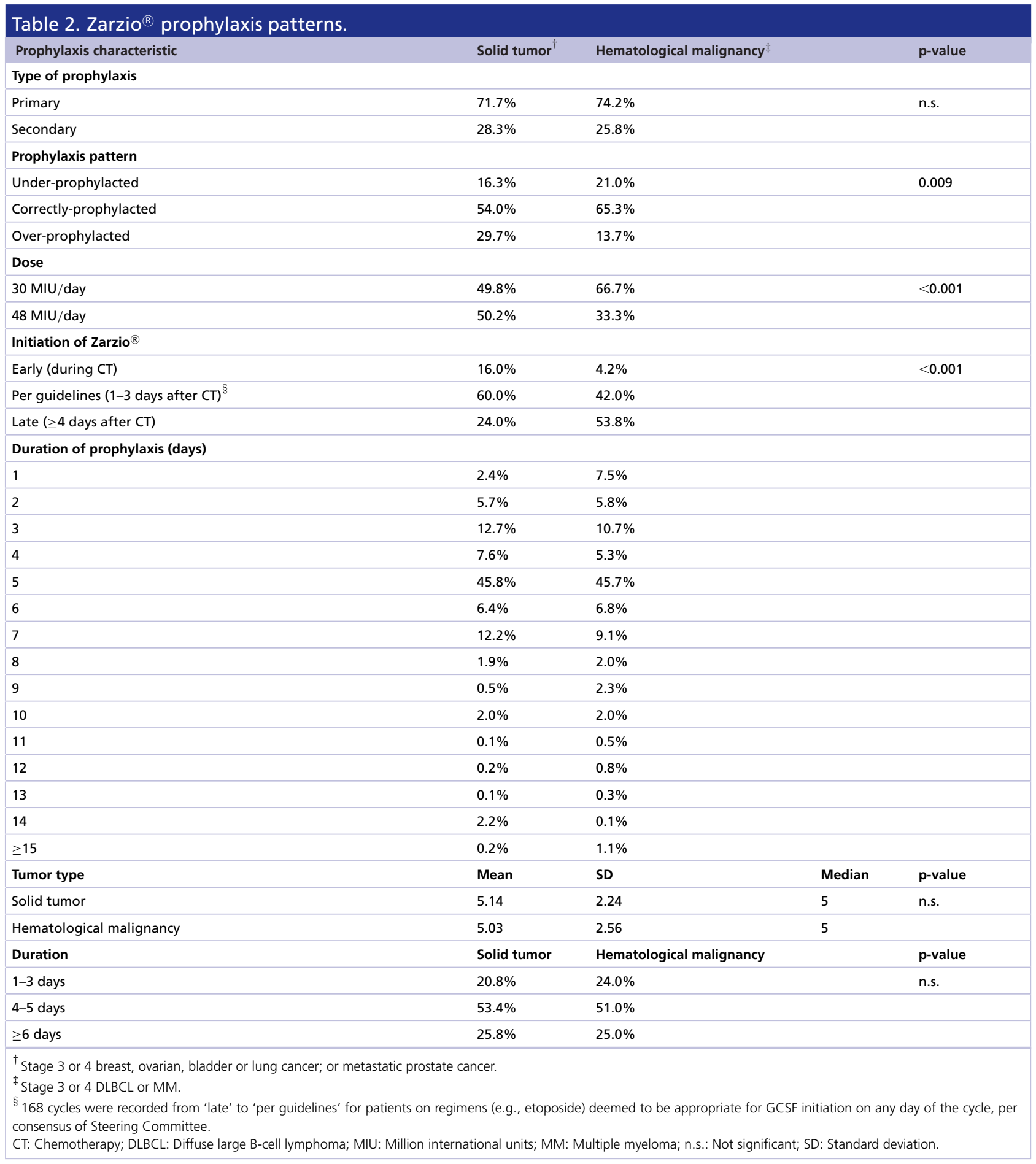

\section{Cycle-level analysis}

Risk of CIN4 during a given cycle in the solid tumor cohort increased with female gender, history of CIN4, CIN1/4 in the previous cycle, in-cycle antibiotic prophylaxis, $48 \mathrm{MIU} /$ day Zarzio ${ }^{\circledR}$ dose, and liver, renal or cardiac disease; but was mitigated with a GIS of 1, indicating Zarzio ${ }^{\circledR}$ initiation in the 24-72-h time window (Supplementary Tables 1Bi \& 2). In-cycle FN risk was a function of ECOG status, history of CIN4 at enrollment and in-cycle antibiotic prophylaxis. CIN/FN hospitalization risk increased with ECOG score, in-cycle antibiotic prophylaxis, history of CIN4 at enrollment and under-prophylaxis but was mitigated by over-prophylaxis. Odds 


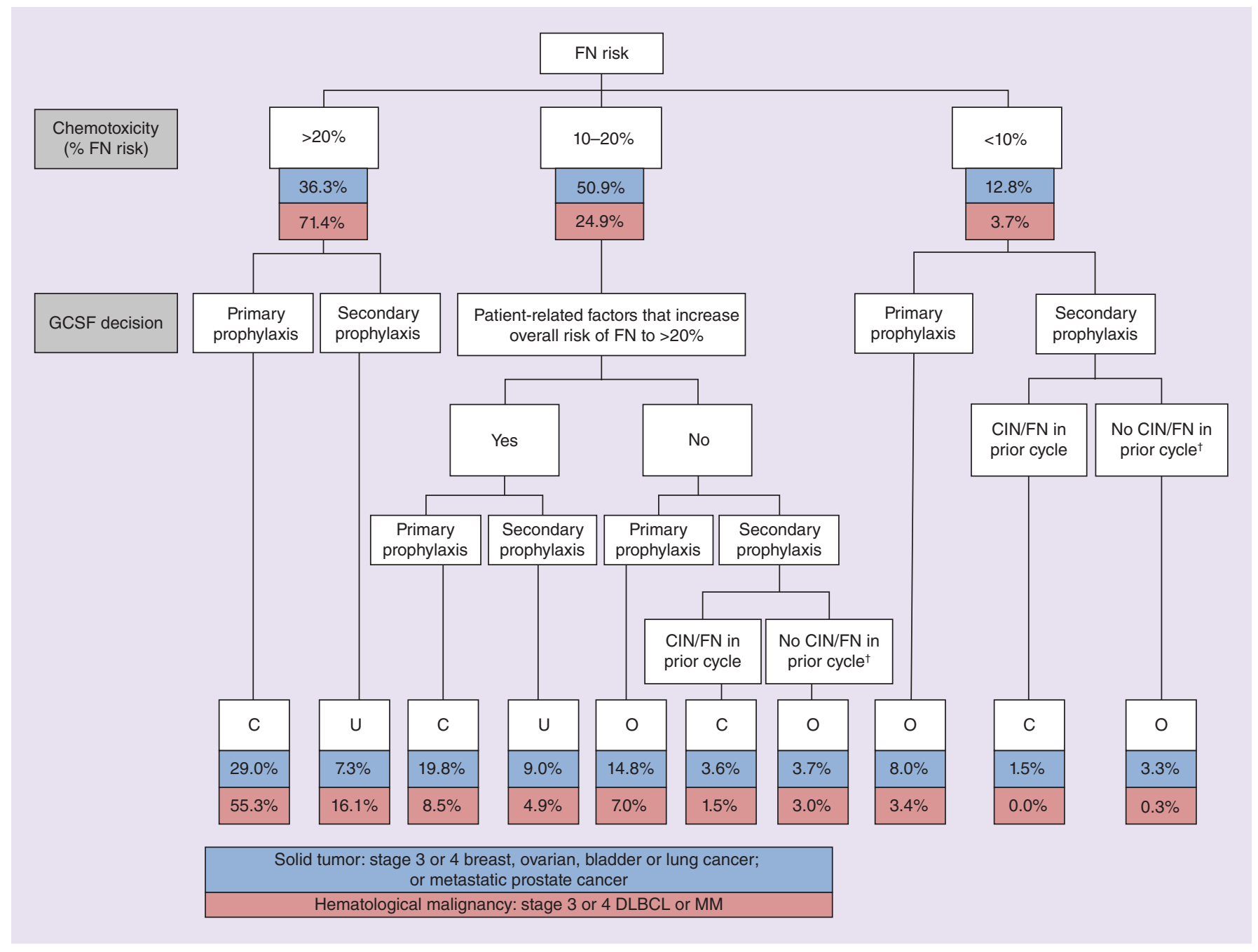

Figure 1. Treatment decision relative to European Organisation for Research and Treatment of Cancer guidelines by cohort. †Secondary prophylaxis started in cycle 2 or later despite no CIN/FN in prior cycle.

C: Correctly-prophylacted; CIN: Chemotherapy-induced neutropenia; DLBCL: Diffuse large B-cell lymphoma; FN: Febrile neutropenia; MM: Multiple myeloma; O: Over-prophylacted; U: Under-prophylacted.

of a chemotherapy disturbance were higher in solid tumor patients with CIN1/4 in a prior cycle as well as certain center-level predictors: center's volume of chemotherapy-treated patients and the center being either an academic or academic-affiliated center; but was attenuated by the centre's volume of cancer patients and number of patients with $\mathrm{FN}$ in the year prior to start of study. The c-statistics ranged from 0.70 to 0.78 .

\section{Predictive risk modeling: hematological malignancy cohort Patient-level analysis}

An ECOG score $\geq 2$ at any time was the only predictor of 'ever' experiencing CIN4 in the hematology cohort (Supplementary Tables 1 Aii \& 2). ECOG score $\geq 2$ also increased odds of FN, as did Zarzio ${ }^{\circledR}$ duration of 4-5 days, compared with duration of 1-3 days, which was associated with decreased risk. No predictors of CIN/FN-related hospitalization, chemotherapy disturbance or composite outcome were retained in the patient-level analyses for hematology patients. The c-statistics for the CIN4 and FN models were 0.58 and 0.76 , respectively.

\section{Cycle-level analysis}

The odds of CIN4 during a given cycle in hematology patients rose if the patient had CIN1/4 in a previous cycle, history of repeated infections, antibiotic prophylaxis during the cycle or $>5 \%$ weight loss in the past month 
(A)

A At the patient level

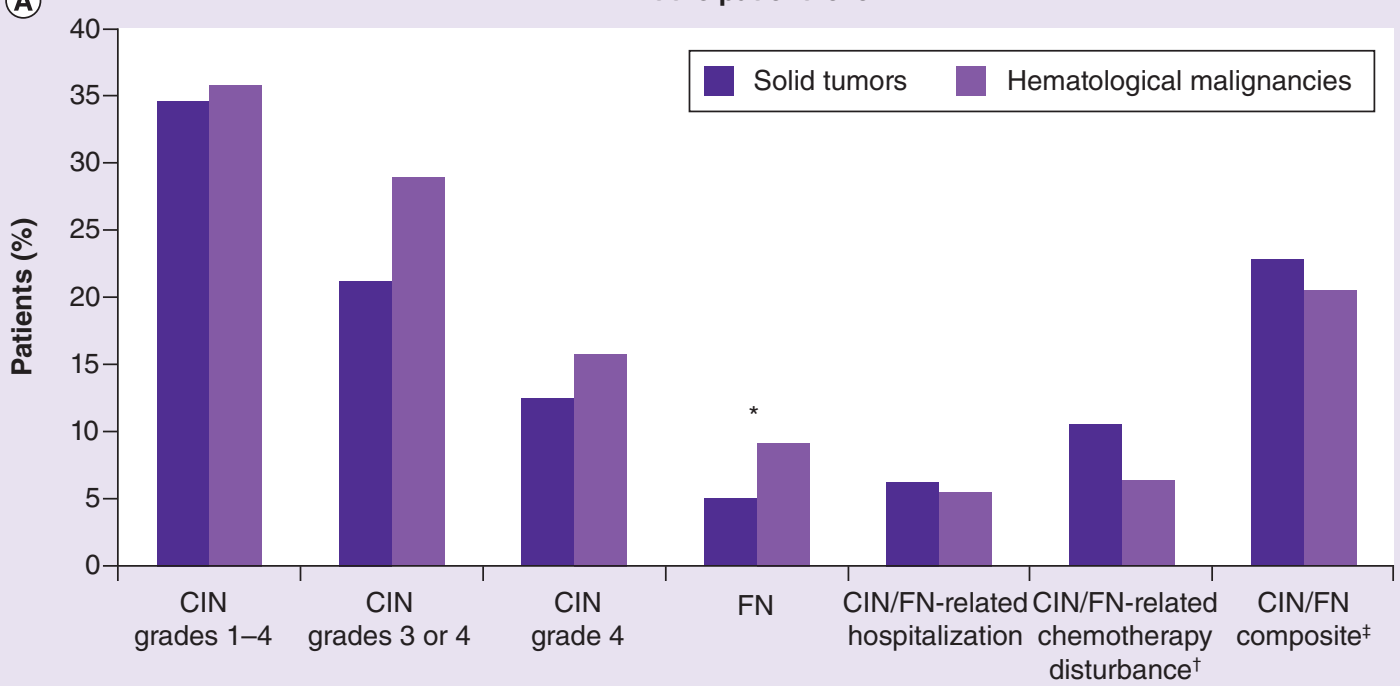

(B)

At the cycle level

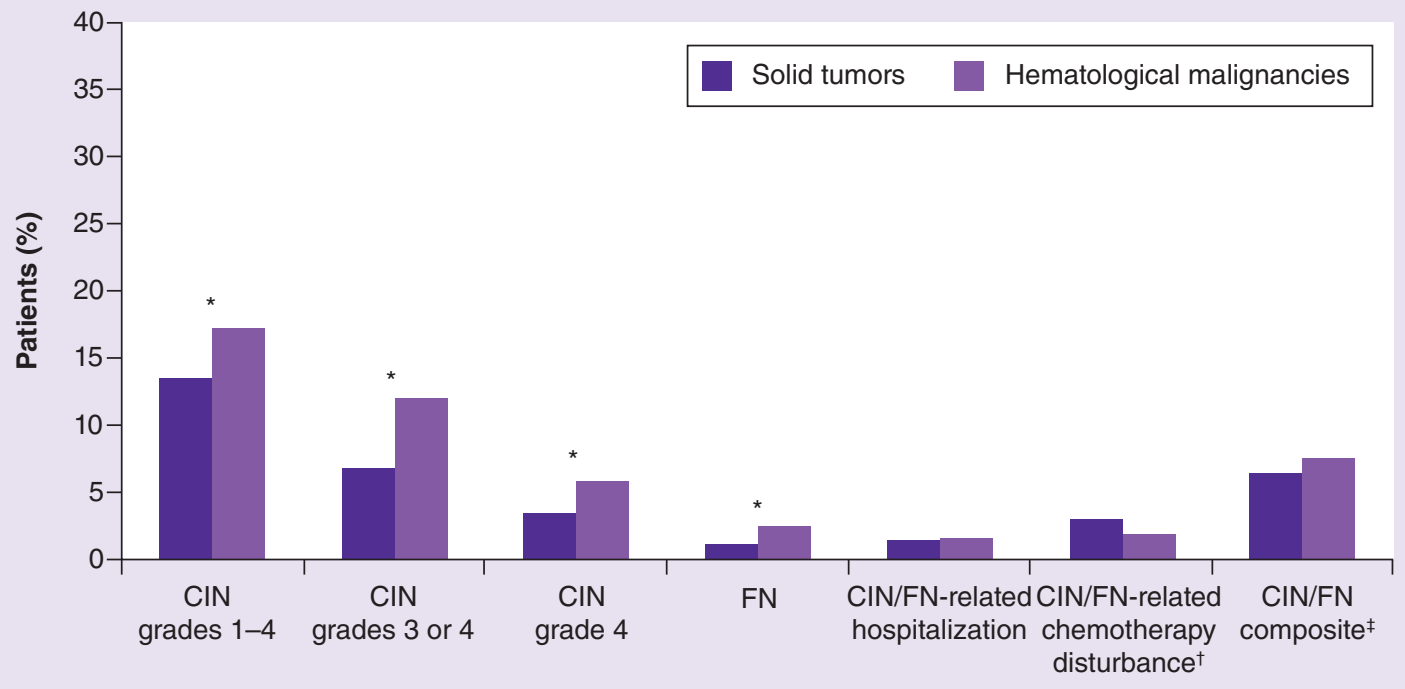

Figure 2. Clinical outcomes by cohort. Solid tumor: stage 3 or 4 breast, ovarian, bladder or lung cancer; or metastatic prostate cancer. Hematological malignancy: stage 3 or 4 diffuse large B-cell lymphoma or multiple myeloma. CIN grades 1-4, grades 3 or 4, grade 4 and FN not mutually exclusive.

†Chemotherapy disturbance includes dose reduction, discontinuation or delay. Measured with one-cycle lag.

\#Includes any occurrence of CIN grade 4, FN, CIN/FN-related hospitalization and/or CIN/FN-related chemotherapy disturbance.

*Difference between solid tumor and hematological malignancy cohorts significant at $p<0.05$.

CIN: Chemotherapy-induced neutropenia; FN: Febrile neutropenia.

(Supplementary Tables 1 Bii \& 2). Odds of FN occurring during a cycle increased with CIN1/4 in a previous cycle and GCSF duration of 4-5 days. Prophylaxis duration of 4-5 days was also associated with higher odds of CIN/FN-related hospitalization. Risk of chemotherapy disturbance increased if CIN1/4 occurred in a previous cycle. Risk for the composite outcome increased with CIN1/4 in a previous cycle, history of repeated infections, antibiotic prophylaxis and 4-5 days of GCSF. Risk reduction was associated with GIS score of 1 (for outcomes CIN4, FN, composite) and 1-3 days of GCSF (FN, hospitalization, composite). The c-statistics ranged from 0.71 to 0.81 . 


\section{Discussion}

A principal finding of this stratified analysis comparing cohorts of solid tumor and hematological malignancy revealed a higher proportion of hematology patients $(9.1$ vs 5.0\%) who experienced FN during the course of chemotherapy, but found no differences between cohorts in rates of CIN of any grade, CIN/FN-related hospitalizations and chemotherapy disturbances, or the composite outcome. While there were proportionately more cycles in which hematology patients experienced CIN of any grade (17.3/13.5\%), grade $3 / 4(12.0 / 6.8 \%)$ and grade 4 $(5.7 / 3.4 \%)$, as well as FN $(2.5 / 1.1 \%)$, there were no differences in cycle-specific hospitalizations or chemotherapy disturbances, or in the composite outcome. Thus, DLBCL and MM patients in our study were more likely to have an FN episode 'ever' during chemotherapy and to have more frequent CIN/FN episodes compared with the solid tumor cohort; yet, despite these higher in-cycle CIN/FN rates, they were not more likely to experience CIN/FN-related hospitalizations or chemotherapy disturbances either within cycles or 'ever' during chemotherapy.

While PRS was similar between cohorts, there were notable differences on some unweighted EORTC-identified risk factors. Hematological patients tended to be older, more often anemic, more impaired in performance status and treated with more myelotoxic chemotherapies and antibiotic prophylaxis. Patients with solid tumors tended to be younger, female and treated mainly with chemotherapy with 10-20\% FN risk; fewer were anemic and they had better performance status; very few required antibiotic prophylaxis; yet, more had advanced disease.

The cohorts also diverged in terms of GCSF prophylaxis patterns. Half of the solid tumor cohort was correctlyprophylacted and another 30\% were over-prophylacted, attributable to their high rate of regimens with 10-20\% FN risk. More hematology than solid tumor patients were under-prophylacted. In $60 \%$ of solid tumor patients, GCSF was initiated within the recommended time window compared with $42 \%$ of hematology patients, largely due to late GCSF initiation in DLBCL patients. Very few hematology patients were started on the day when chemotherapy was completed; however, one in six patients with solid tumor were.

The modeling results provide additional insights into neutropenia prophylaxis in patients with solid tumors and hematological malignancies (Supplementary Table 2). Across patient- and cycle-level analyses, predictors of poor outcomes common to both cohorts included concomitant antibiotic prophylaxis, ECOG performance status greater than or equal to two any time during the study and CIN of any grade in a prior cycle; while a GIS score of 1 , indicating Zarzio ${ }^{\circledR}$ initiation within the $24-72$-h window, was a common strong predictor of better outcomes. Hematology patients were at greater risk for poor outcomes if they had a history of repeated infections, had weight loss of $>5 \%$ in the preceding month or received 4-5 days as opposed to 1-3 days of GCSF support (suggesting perhaps that their physician was quite concerned about CIN/FN). Their likelihood of better outcomes was a function of shorter (1-3 days) prophylaxis duration (perhaps indicating less physician concern about CIN/FN), and if they had also received radiotherapy. Among patients with solid tumors, poor outcomes were especially more likely with under-prophylaxis and history of CIN4, and, though to a lesser extent, if female, with liver/renal/cardiovascular disease, receiving secondary prophylaxis and/or treated with the $48 \mathrm{MIU} /$ day dose (the latter indicating, perhaps, a more difficult-to-prophylact patient). The strongest predictor of better outcomes in patients with solid tumors was over-prophylaxis.

These findings translate into specific clinical recommendations; some general to GCSF prophylaxis, some specific to each cohort. In general, and regardless of whether patients have hematological malignancies or solid tumors, clinicians should follow evidence-based guidelines and assess patients' CIN/FN risk at the start of chemotherapy and at the beginning of each cycle. They should be particularly attentive to patients with poor or worsening performance status (ECOG greater than or equal to two), those with CIN of any grade in a prior cycle and patients requiring antibiotic prophylaxis; while initiating GCSF support in the recommended 24-72-h window after chemotherapy.

In addition, for hematology patients, or at least for the patients with DLBCL or MM studied here, clinical vigilance is indicated in patients with history of repeated infections; weight loss $>5 \%$ over 1 month, especially with a parallel decline in performance status; and those whose clinicians believe might be at greater risk of $\mathrm{CIN} / \mathrm{FN}$ and related complications (as suggested by the decision to prophylact longer than just a few days). In fact, one major implication of our findings is that hematology patients require defensive clinical attention to prevent hematology-specific risk factors from eliciting poor outcomes.

In patients with solid tumors, poor outcomes can be prevented by avoiding under-prophylaxis and secondary prophylaxis and, in fact, by over-prophylacting patients, especially if female and/or with liver/renal/cardiovascular 
disease. Considering the higher $48 \mathrm{MIU} /$ day Zarzio $^{\circledR}$ dose may suggest to clinicians that they are indeed concerned about CIN/FN complications.

In addition to limitations identified in our prior reports [21,22], the present analyses warrant some caution. Our hematology cohort was limited to patients with DLBCL and MM. Future studies should include a broader range of hematological malignancies typically treated with myelotoxic chemotherapy as well as rare solid tumors such as sarcomas. Concomitant antibiotic prophylaxis in hematology patients may be less common in daily practice than it was in our study and limited mainly in cases where a high neutropenic risk is expected or as pneumocystis pneumonia prophylaxis in patients treated with CD20 antibodies. The preponderance of female patients in the solid tumor cohort, attributable to the breast cancer population, may point at a selection bias in our study.

\section{Conclusion}

In our study, no differences were found between the solid tumor and hematological malignancy cohorts in rates of patients ever experiencing CIN of any grade (except for FN) even though hematology patients experienced CIN/FN episodes more frequently, likely due to their higher rate of chemotherapies with $\geq 20 \%$ FN risk. Yet, these in-cycle differences did not translate into higher rates of CIN/FN-related hospitalizations or chemotherapy disturbances either within cycles or at the patient level.

Our stratified analyses underscore the importance of assuring adequate GCSF support in both hematology and solid tumor patients to prevent CIN/FN and related hospitalizations and chemotherapy disturbances. In general, special attention is warranted for all patients with poor performance status, those requiring antibiotic prophylaxis and those with prior CIN episodes. Timely initiation of GCSF support is paramount. Careful attention to risk factors is indicated while prophylacting in accordance with guidelines; under-prophylaxis should be avoided, especially in patients presenting with EORTC-identified risks.

\section{Summary points}

- Chemotherapy-induced neutropenia (CIN) and febrile neutropenia (FN) are frequent, and potentially life threatening, complications of myelotoxic chemotherapy, which can result in hospitalization or chemotherapy disturbance.

- Standard of care is prophylaxis with GCSF based on the regimen's myelotoxicity and patient risk factors; both standard or pegylated formulations of filgrastim have demonstrated efficacy in prevention and reduction of severity and duration of CIN/FN.

- GCSF is indicated for patients with solid tumors as well as those with lymphoproliferative disorders; however, use in other hematological settings is unclear.

- The prospective, real-world, observational MONITOR-GCSF study, conducted in 140 centers in 12 European countries, evaluated patients with solid tumors (breast, ovarian, bladder or lung; $77.2 \%$ ) or hematological malignancies (diffuse large B-cell lymphoma or multiple myeloma; 22.8\%) receiving biosimilar filgrastim.

- Significantly $(p=0.009)$ more patients were over-prophylacted and fewer were correctly-prophylacted in the solid tumor cohort compared with the hematology cohort.

- In the solid tumor cohort, $60.0 \%$ of patients initiated biosimilar filgrastim in the recommended timeframe (i.e., day 1-3), compared with $53.8 \%$ of hematology patients being initiated late (day 4 or later).

- At the cycle level, patients with hematological malignancies had higher rates of CIN (all grades) and FN than the solid tumor cohort, but no differences in hospitalization or chemotherapy disturbance.

- No differences between cohorts were found in patient-level outcomes ('ever' during study), except for FN $(9.1 \%$ in hematological patients vs $5.0 \%$ ).

- Across analyses at both the patient and cycle levels, predictors of poor outcomes in both the hematology and solid tumor cohorts included concomitant antibiotic prophylaxis, Eastern Cooperative Oncology Group performance status greater than or equal to two at any time during the study, and CIN of any grade in a prior cycle. Conversely, a predictor of better outcomes in both cohorts was a GCSF initiation score of 1, indicating biosimilar filgrastim initiation within the day 1-3 window.

- This analysis illustrates the importance of prophylaxis of both hematology and solid tumor patients with adequate and timely GCSF support to reduce the risk of CIN/FN and related hospitalizations and chemotherapy disturbances. Extra attention is necessary for patients at high risk, especially those with poor performance status, those requiring antibiotic prophylaxis and those with prior CIN episodes. 
Financial \& competing interests disclosure

This work was supported by Hexal AG/Sandoz International GmbH. Sponsor participated in the development of the protocol, implementation of the study, analysis and interpretation of the results, review of the manuscript for scientific content and the decision to submit the manuscript for publication. M Aapro, C Bokemeyer, H Ludwig, P Gascón and M Boccadoro received honoraria for presentations and Advisory Board meetings from Sandoz Biopharmaceuticals. M Aapro also serves on Speakers Bureaus for Amgen, Pfizer, Teva, Kyowa Kirin and Roche. A Krendyukov was an employee of Hexal AG at the time of manuscript development and submission. K Denhaerynck, I Abraham and K MacDonald are affiliated with Matrix45. By company policy, they cannot hold equity in sponsor organizations and cannot receive direct personal benefits, financial or other, from sponsor organizations. Matrix45 provides similar services to other biopharmaceutical companies without exclusivity constraints. The authors have no other relevant affiliations or financial involvement with any organization or entity with a financial interest in or financial conflict with the subject matter or materials discussed in the manuscript apart from those disclosed.

Editorial support was provided by C McGown of Spirit Medical Communications Group Ltd, supported by Sandoz GmbH, Kundl, Austria.

Ethical conduct of research

All procedures performed in studies involving human participants were in accordance with the ethical standards of the institutional and/or national research committees and with the 1964 Helsinki Declaration and its later amendments or comparable ethical standards. All patients consented to treatment according to institutional guidelines.

Open access

This work is licensed under the Attribution-NonCommercial-NoDerivatives 4.0 Unported License. To view a copy of this license, visit http://creativecommons.org/licenses/by-nc-nd/4.0/

Data sharing statement

The authors certify that this manuscript reports the secondary analysis of clinical trial data that have been shared with them, and that the use of this shared data is in accordance with the terms (if any) agreed upon their receipt. The source of this data is: NCT01459653.

\section{References}

Papers of special note have been highlighted as: • of interest; $\bullet \bullet$ of considerable interest

1. Lalami Y, Paesmans M, Muanza F et al. Can we predict the duration of chemotherapy-induced neutropenia in febrile neutropenic patients, focusing on regimen-specific risk factors? A retrospective analysis. Ann. Oncol. 17, 507-514 (2006).

2. Lalami Y, Paesmans M, Aoun M et al. A prospective randomized evaluation of G-CSF or G-CSF plus oral antibiotics in chemotherapy-treated patients at high risk of developing febrile neutropenia. Support Care Cancer 12, 725-730 (2004).

3. Lyman GH, Lyman CH, Agboola O. Risk models for predicting chemotherapy-induced neutropenia. Oncologist 10, 427-437 (2005).

4. Lyman GH, Dale DC, Culakova E et al. The impact of the granulocyte colony-stimulating factor on chemotherapy dose intensity and cancer survival: a systematic review and meta-analysis of randomized controlled trials. Ann. Oncol. 24, 2475-2484 (2013).

5. Lyman GH, Abella E, Pettengell R. Risk factors for febrile neutropenia among patients with cancer receiving chemotherapy: a systematic review. Crit. Rev. Oncol. Hematol. 90, 190-199 (2014).

6. Aapro MS, Bohlius J, Cameron DA et al. 2010 update of EORTC guidelines for the use of granulocyte-colony stimulating factor to reduce the incidence of chemotherapy-induced febrile neutropenia in adult patients with lymphoproliferative disorders and solid tumours. Eur. J. Cancer 47, 8-32 (2011).

- The latest guidance on prevention and management of chemotherapy-induced febrile neutropenia (CIN/FN) using granulocyte-colony stimulating factor outlined by the European Organisation for Research and Treatment of Cancer.

7. Smith TJ, Bohlke K, Lyman GH et al. Recommendations for the use of WBC growth factors: American Society of Clinical Oncology clinical practice guideline update. J. Clin. Oncol. 33, 3199-3212 (2015).

- The latest guidance on prevention and management of CIN/FN using GCSF outlined by the American Society of Clinical Oncology.

8. National Comprehensive Cancer Network. NCCN clinical practice guidelines in oncology. Myeloid growth factors. Version 2. (2018). www.nccn.org/professionals/physician_gls/PDF/myeloid_growth.pdf

- The latest guidance on the use of myeloid growth factors, including GCSF, to reduce the incidence of CIN/FN outlined by the National Comprehensive Cancer Network.

9. Wingard JR, Elmongy M. Strategies for minimizing complications of neutropenia: prophylactic myeloid growth factors or antibiotics. Crit. Rev. Oncol. Hematol. 72, 144-154 (2009). 
10. Gridelli C, Aapro M, Barni S et al. Role of colony stimulating factors (CSFs) in solid tumours: results of an expert panel. Crit. Rev. Oncol. Hematol. 63, 53-64 (2007).

11. Klastersky J, Awada A. Prevention of febrile neutropenia in chemotherapy-treated cancer patients: pegylated versus standard myeloid colony stimulating factors. Do we have a choice? Crit. Rev. Oncol. Hematol. 78, 17-23 (2011).

12. Klastersky J, Awada A, Paesmans M, Aoun M. Febrile neutropenia: a critical review of the initial management. Crit. Rev. Oncol. Hematol. 78, 185-194 (2011).

13. Cooper KL, Madan J, Whyte S, Stevenson MD, Akehurst RL. Granulocyte colony-stimulating factors for febrile neutropenia prophylaxis following chemotherapy: systematic review and meta-analysis. BMC Cancer 11, 404 (2011).

14. Lyman G, Dale D, Wolff DA et al. Acute myeloid leukemia or myelodysplastic syndrome in randomized controlled clinical trials of cancer chemotherapy with granulocyte colony-stimulating factor: a systematic review. J. Clin. Oncol. 28, 2914-2924 (2010).

15. Giebel S, Thomas X, Hallbook $\mathrm{H}$ et al. The prophylactic use of granulocyte-colony stimulating factor during remission induction is associated with increased leukaemia-free survival of adults with acute lymphoblastic leukaemia: a joint analysis of five randomised trials on behalf of the EWALL. Eur. J. Cancer 48, 360-367 (2012).

16. Wheatley K, Goldstone AH, Littlewood T, Hunter A, Burnett AK. Randomized placebo-controlled trial of granulocyte colony stimulating factor (G-CSF) as supportive care after induction chemotherapy in adult patients with acute myeloid leukaemia: a study of the United Kingdom Medical Research Council Adult Leukaemia Working Party. Br. J. Haematol. 146, 54-63 (2009).

17. National Comprehensive Cancer Network. NCCN clinical practice guidelines in oncology. Myelodysplastic syndromes. Version 2 (2018). www.nccn.org/professionals/physician_gls/pdf/mds.pdf

18. National Comprehensive Cancer Network. NCCN clinical practice guidelines in oncology. Acute myeloid leukemia. Version 2 (2018). www.nccn.org/professionals/physician_gls/pdf/aml.pdf

19. Gascón P, Aapro M, Ludwig H et al. Background and methodology of MONITOR-GCSF, a pharmaco-epidemiological study of the multi-level determinants, predictors, and clinical outcomes of febrile neutropenia prophylaxis with biosimilar granulocyte-colony stimulating factor filgrastim. Crit. Rev. Oncol. Hematol. 77, 184-197 (2011).

-• Context, design and methods of the pan-European, prospective, observational, pharmaco-epidemiological MONITOR-GCSF study, which aimed to assess the real-world multilevel factors and outcomes of biosimilar filgrastim in the prophylaxis of FN in cancer patients receiving chemotherapy.

20. Gascón P, Aapro M, Ludwig H et al. Update on the MONITOR-GCSF study of biosimilar filgrastim to reduce the incidence of chemotherapy-induced febrile neutropenia in cancer patients: protocol amendments. Crit. Rev. Oncol. Hematol. 77, 198-200 (2011).

21. Gascón P, Aapro M, Ludwig H et al. Treatment patterns and outcomes in the prophylaxis of chemotherapy-induced (febrile) neutropenia with biosimilar filgrastim (the MONITOR-GCSF study). Support Care Cancer 24, 911-925 (2016).

-. Results from the entire cohort $(n=1447)$ of MONITOR-GCSF, a pan-European, prospective, observational, pharmaco-epidemiological study examining real-world treatment patterns and outcomes of biosimilar filgrastim for prophylaxis of CIN/FN.

22. Aapro M, Ludwig H, Bokemeyer P et al. Predictive modeling of the outcomes of chemotherapy-induced (febrile) neutropenia prophylaxis with biosimilar filgrastim (MONITOR-GCSF study). Ann. Oncol. 27, 2039-2045 (2016).

-. 'Real-world' models of predictors of patients experiencing an adverse neutropenic event based on data from the international, prospective, observational MONITOR-GCSF study; these models provide oncologists with a method of anticipating such clinical developments.

23. Twisk JWR. Applied Longitudinal Data Analysis for Epidemiology: A Practical Guide (2nd Edition). Cambridge University Press, Cambridge, UK (2013). 
